\title{
Telemedicine in Primary Care by Dr Maria Leung
}

\author{
Maria Leung* \\ Consultant in Department of Family Medicine, Prince of Wales Hospital, New Territories East Cluster, Hospital Authority, \\ Hongkong
}

*Corresponding author: Maria KW Leung, Consultant in Department of Family Medicine, Prince of Wales Hospital, New Territories East Cluster, Hospital Authority, Hongkong

\begin{tabular}{lll}
\hline ARTICLE INFO & & Abstract \\
\cline { 1 - 2 } $\begin{array}{l}\text { Received: 幽 May 11, } 2020 \\
\text { Published: }\end{array}$ & & $\begin{array}{l}\text { Citation: Maria Leung. Telemedicine in Primary Care by Dr Maria Leung. Biomed J Sci } \\
\text { \& Tech Res 28(1)-2020. BJSTR. MS.ID.004580 }\end{array}$ \\
\hline
\end{tabular}

\section{Opinion}

The term "Telemedicine" was first used in the 1970s, meaning "healing at a distance. In fact, the use of this concept was mentioned as early as in 1960's. One such example was the provision of telemedicine to astronauts on space missions in National Aeronautics and Space Administration (NASA) [1]. Later in 1998, World Health Organisation (WHO) adopted the description as "The delivery of health care services, where distance is a critical factor, by all health care professionals using information and communication technologies for the exchange of valid information for diagnosis, treatment and prevention of disease and injuries, research and evaluation, and for the continuing education of health care providers, all in the interests of advancing the health of individuals and their communities"[2]. With the introduction of internet and advance in technology, telemedicine has become more user-friendly and many trials have been done to assess its effectiveness on health care and its impact on patients and healthcare professionals. A Cochrane database review on telemedicine was published in 2015, including 93 trials and about 22000 patients [3].

Most of these studies were to provide monitoring of chronic illnesses, treatment or rehabilitation, education and advice on selfmanagement, real time assessment of clinical status, screening, and specialist consultations for diagnosis and treatment decisions. Regarding impact on health care outcomes, the review found that telemedicine may improve glucose control in diabetics. Some studies showed lower blood pressure in patients with hypertension via monitoring of telemedicine, and some studies looked at the effect of telemedicine on patients with mental health and substance abuse problems and did not find any differences in the effect of therapy given via video-conferencing when compared to face-toface delivery. One study in 2017 showed that the evaluation time by dermatologist was markedly shortened from 70 days to 0.5 day after the use of tele dermatology [4]. As majority of patients in primary care have chronic illnesses such as hypertension, diabetes mellitus and mental health problems, the results from these studies support the use of telemedicine in primary care. Not only could it improve healthcare outcomes of those with chronic illnesses, but it could also help to reduce referral rates and waiting time. Indeed, telemedicine should be one of the ways for future development in primary care if we have to manage the increasing volume of patients due to the ageing population.

Due to COVID, many schools have been closed and their teachings have been switched to web based. This also applies to seminars, meetings, or overseas conferences for our healthcare professionals. In fact, to our surprise, such online teachings via Google meet, Zoom, Webinar have been welcome by many due to lack of limitations due to geographical reasons and time saving in travelling. In the past, the contingency plan, in case of bad weather, would be to cancel the seminar or lecture. But now, the contingency plan can be to convert to online teaching without wasting the effort from the speaker or the organizing team. Using internet system for direct video consultation between patient and doctor has certainly marked its importance in healthcare provision. Future advanced technology should focus on better electronic health record system, data accuracy and information integration between patients and our healthcare system. For instance, direct entry of their home blood pressure or histix readings into their electronic clinical 
records, thus allowing doctor to easily monitor their control, would be essential in enhancing the efficiency in primary care.

However, one must bear in mind that advanced technology alone cannot improve the healthcare outcomes. The success of telemedicine should comprise of careful planning in management and well written protocols, with accurate reminders or prompting for patient and health care providers so that timely intervention can be carried out. In primary care settings, part of the therapeutic effect would be based on the trustful relationship between the family physician and the patient. So, one must not forget that such relationship should be established professionally before responsible telemedicine service is to take place [5]. As physicians, we should also be aware of the ethical issues related to the use of telemedicine such as personal data privacy and confidentiality. In this current tough time, while we are all busy and tired in fighting against the coronavirus, we, as healthcare professionals, must stay positive and grasp each opportunity to learn and create new way to care for our community.

ISSN: 2574-1241

DOI: $10.26717 /$ BJSTR.2020.28.004580

Maria Leung. Biomed J Sci \& Tech Res

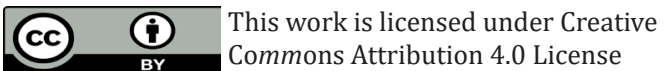

Submission Link: https://biomedres.us/submit-manuscript.php

\section{Acknowledgement}

None.

\section{Conflict of Interest}

None.

\section{References}

1. History of telemedicine. Md Portal.

2. WHO (1997) A health telematics policy in support of WHO's Health-ForAll strategy for global health development, report of the WHO group consultation on health telematics, 11-16 December, Geneva, 1007, Geneva, World Health Organisation, 1997.

3. Flodgren G, Rachas A, Farmer AJ, Inzitari M, Shepperd S, et al. (2015) Interactive telemedicine: effects on professional practice and health care outcomes. Cochrane Database of Systematic Reviews 9: CD002098.

4. Carter, Shauna Goldman, Kristen Anderson, Xiaxiao Li, Linda S Hynan, et al. (2017) Creation of an internal teledermatology Store-and-Forward System in an existing electronic health record. JAMA Dermatol 153(7): 644-650.

5. Daniel H, Sulmasy LS (2015) Policy recommendations to guide the use of telemedicine in primary care settings: An American College of Physicians Position Paper. Ann Intern Med 163: 787-789.

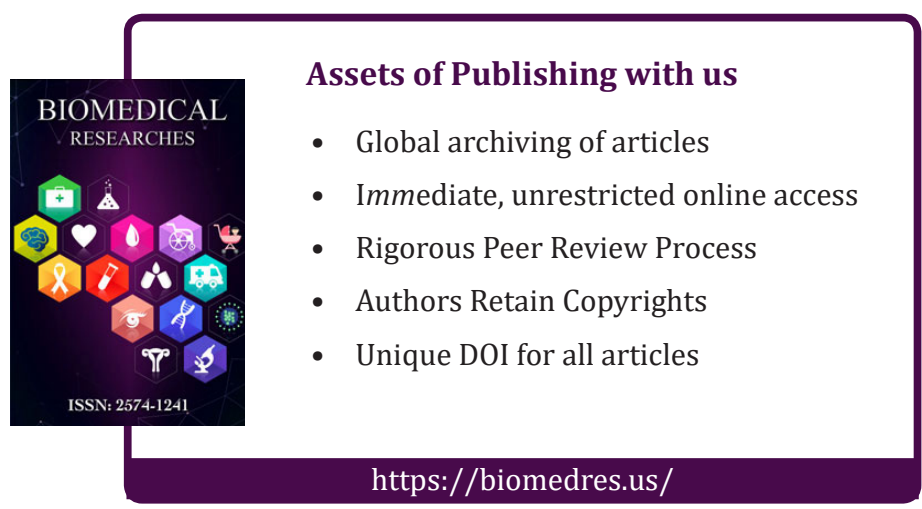

